\title{
Incidencia de la gestión universitaria en la deserción estudiantil de las universidades públicas en Chile
}

\author{
Incidence of university management on student dropout in public universities in Chile \\ Daniela Sanhueza Gutiérrez \\ Andrea King-Domínguez \\ Luis Améstica-Rivas
}

\begin{abstract}
RESUMEN
La deserción estudiantil afecta a los sistemas educativos a nivel global y Chile no es la excepción, especialmente en el sector universitario. No existe una única razón que lleve a los estudiantes a desertar, sino más bien es un fenómeno multicausal, las condiciones de cada institución toman relevancia al intentar explicar este fenómeno. En este contexto, el objetivo de esta investigación es identificar y analizar los factores institucionales que explican la deserción estudiantil de las universidades chilenas. A partir de una muestra de 28 universidades llamadas públicas, integrada por instituciones privadas y estatales, se aplicaron tres tipos de regresiones de tipo panel de datos sobre variables explicativas para el periodo 2016-2018. En un primer análisis se evidenció que la tasa promedio de deserción estudiantil es mayor en las universidades estatales en comparación con las privadas. Del mismo modo se logró evidenciar que, a mayor cantidad de docentes con postgrado, a mayor número de metros cuadrados construidos de salas de estudios, laboratorios y salas de clases por estudiantes, y a mayor número de bibliotecas académicas, menor es la tasa de deserción estudiantil. Estos resultados podrían ser interesantes para la definición de políticas institucionales.
\end{abstract}

Palabras clave: fracaso escolar, universidad, enseñanza y formación, deserción universitaria.

\section{ABSTRACT}

Student dropout affects education systems globally and Chile is no exception, especially in the university sector. There is no single reason that leads students to drop out, but rather it is a multicausal phenomenon, conditions of each institution take relevance when trying to explain this phenomenon. In this context, the objective of this research is to identify and analyze the institutional factors that explain student dropout in Chilean universities. Starting from a sample of 28 so-called public universities, integrated by private and state institutions, three types of panel-type regressions of data on explanatory variables were applied for the period 2016-2018. In a first analysis, it was evidenced that the average student dropout rate is higher in state universities compared to private ones. Similarly, it was found that the greater the number of teachers with postgraduate degrees, the greater the number of square meters of study rooms, laboratories, and classrooms per student, and the greater the number of academic libraries, the lower the student dropout rate. These results could be interesting for the definition of institutional policies.

Keywords: academic failure, university, education and training, university dropout. 


\section{INTRODUCCIÓN}

Las universidades tienen un rol importante en el desarrollo socioeconómico de un país, ya que se centran, dentro de una diversidad de campos, en la transmisión de conocimientos y el fomento de la investigación. Por esta razón, en el largo plazo la principal ventaja comparativa de los países para el progreso económico será el talento de su población. Esto explicaría la prioridad que las naciones de Asia del este y los países escandinavos le han dado al desarrollo de su sistema de educación en todos niveles (Martí, Puertas y Calafat, 2014; Salmi, 2014). De esta manera, la educación superior aporta a las personas elementos y posibilidades indispensables para el desarrollo personal y laboral de los estudiantes, y de la sociedad en su conjunto.

Sobre la base de lo anterior, no es de extrañar que en Chile se considere que la educación superior es un derecho y que debe estar al alcance de todas las personas, de acuerdo a sus capacidades y méritos (BCN, 2018). La relevancia que tiene la educación superior en Chile se ve reflejada en el esfuerzo que ha hecho la sociedad para apoyar a todas las personas que requieran de financiamiento para continuar o realizar sus estudios de educación superior. Es así que a partir del año 2016 comenzó a regir la política pública impulsada por el Estado concerniente a otorgar gratuidad en la educación superior, con la finalidad de crear un sistema de educación más igualitario, inclusivo y capaz de garantizar el acceso y permanencia de los estudiantes en la educación superior, independientemente de su condición socioeconómica (MINEDUC, 2016).

En Chile hay 149 instituciones de educación superior, las que incluyen a 59 universidades. Entre estas últimas, 30 (18 estatales y 12 privadas) integran el Consejo de Rectores de las Universidades Chilenas (CRUCH) y son definidas como tradicionales

Daniela Sanhueza Gutiérrez. Investigadora de la Facultad de Ciencias Empresariales, Universidad del Bío-Bío, Concepción, Chile. Es licenciada en Ciencias Administrativas y magíster en Gestión de Empresas. Correo electrónico: daniela.sanhueza1501@ alumnos.ubiobio.cl. ID: https://orcid.org/0000-0001-8200-0976.

Andrea King-Domínguez. Académica del Departamento de Economía y Finanzas de la Facultad de Ciencias Empresariales, Universidad del Bío-Bío, Concepción, Chile. Es doctora en Administración y Dirección de Empresas por la Universidad Politécnica de Cataluña; magíster en Administración mención Finanzas por la Universidad de Chile e investigadora asociada al grupo de investigación en Dirección Universitaria de la Universidad Politécnica de Cataluña, España. Ha sido directora de Recursos Humanos de la misma universidad y ha ejercido distintos cargos académicos. Posee más de 25 publicaciones. Correo electrónico: aking@ubiobio.cl. ID: https://orcid.org/0000-0002-1063-4336.

Luis Améstica-Rivas. Académico del Departamento de Gestión Empresarial de la Facultad de Ciencias Empresariales, Universidad del Bío-Bío, Chillán, Chile. Es doctor en Administración y Dirección de Empresas de la Universidad Politécnica de Cataluña; magíster en Administración mención Finanzas por la Universidad de Chile y actualmente investigador del grupo de investigación Dirección Universitaria de la Universidad Politécnica de Cataluña, España. Participa en redes de investigación en Europa, México y Chile. Profesor de finanzas y proyectos en distintas universidades chilenas en pre y postgrado. Ha sido gerente de finanzas y de empresas, consultor y directivo universitario. Posee más de 40 publicaciones. Correo electrónico: lamestica@ ubiobio.cl. ID: https://orcid.org/0000-0003-0482-0287. 
y con plena autonomía. Todas estas universidades son consideradas líderes en investigación y postgrado, además de contar con acreditación institucional, otorgada por la Comisión Nacional de Acreditación (CRUCH, 2021; Mineduc, 2020).

En este contexto, al igual que en otros países, la deserción estudiantil es un problema. Según Kim y Kim (2018), este fenómeno no solo afecta al individuo que abandona, sino también a la universidad y a la sociedad. De manera similar, Rodríguez y Zamora (2014) explican que, desde el punto de vista de la sociedad, incide negativamente en los índices de pobreza, aumenta el desempleo, disminuye el aporte intelectual e incrementa el costo de la educación. En relación con lo institucional, la deserción afecta el cumplimiento de la misión de la organización y baja los índices de eficiencia, calidad e ingreso. Por último, en el ámbito personal se percibe la sensación de fracaso, pérdida de oportunidades laborales, impactando de igual forma en términos individuales y familiares.

Fundado en lo anterior, esta investigación busca identificar y analizar los factores institucionales que explican la deserción estudiantil de las universidades chilenas.

\section{MARCo TEÓRICO}

El concepto de "deserción" ha sido definido por diversos autores y ha estado en constante evolución a través del tiempo. Inicialmente, Tinto (1975) definió el concepto de deserción como un proceso de abandono voluntario o forzoso de la carrera en que se matricula un estudiante, por la influencia positiva o negativa de circunstancias internas o externas al alumno. Posteriormente, Himmel (2002) precisó que es el abandono prematuro de un programa de estudios antes de alcanzar el título o grado, y considera un tiempo suficientemente largo como para descartar la posibilidad de que el estudiante se reincorpore.

Es por esto que el estudio de la deserción estudiantil en las universidades es un tema de gran relevancia, siendo considerado como un indicador de calidad de la gestión universitaria (Cabrera, Bethencourt, Alvarez y González, 2006). De acuerdo con Tapasco, Ruiz, Osorio y Ramírez (2019) existiría consenso en aceptar que la tasa de abandono es un indicador negativo de la calidad de la universidad, pues se entiende que no estaría proporcionando los medios necesarios para que los estudiantes, que lo intentaron, obtuvieran el título esperado.

En cuanto a las razones que llevan a los estudiantes a desertar, se ha determinado que existen múltiples motivos que la ocasionan, de forma que es considerada la deserción como un fenómeno multicausal. Entre los factores que llevan a un estudiante universitario a desertar se podría mencionar la baja calidad de la enseñanza, las dificultades financieras, la insatisfacción con los requisitos o las regulaciones, los cambios en los planes de estudio y las bajas calificaciones (Tinto, 1975; Smart, 1997; Munizaga, Cifuentes y Beltrán, 2018). 
Para autores como Tinto (1975) y Himmel (2002), las razones que llevan a un estudiante de la educación superior a abandonar pueden clasificarse como involuntarias o voluntarias. En cuanto a las razones voluntarias de abandono de los estudios, Larsen, Sommersel y Larsen (2013) dicen que ocurrirían cuando el alumno decide dejar sus estudios para cursar otra materia o cambiar de universidad, o debido a circunstancias externas, como dificultades financieras o problemas familiares o personales.

Por otra parte, la deserción involuntaria sería consecuencia de una decisión institucional fundada por los reglamentos universitarios, es decir, cuando el estudiante no cumple con los estándares académicos y las demandas dentro de la universidad, lo que implica una transferencia (Himmel, 2002; Larsen, Sommersel y Larsen, 2013).

Las razones de la deserción estudiantil, sean voluntarias o involuntarias, podrían ser clasificadas en distintas categorías. Kehm, Larsen y Soomersel (2020), sobre la base de una revisión bibliográfica, explicaron que las razones de la deserción estudiantil podrían clasificarse en siete grupos.

- Antecedentes sociodemográficos del alumno. En este grupo se considerarían los niveles educacional y ocupacional de los padres.

- Las razones referentes a los rendimientos previos (rendimiento en la educación media, enfoque y tipo del establecimiento de la educación media).

- Requisitos de admisión e información.

- Esfuerzos personales y motivaciones para estudiar, que pueden ser extrínsecas (interés en el trabajo futuro) e intrínsecas (interés en la materia).

- Integración académica en la universidad, lo que se relaciona a características objetivas (rendimiento académico) y subjetivas (progreso autopercibido o la integración e interacción con el personal académico).

- Integración social del estudiante en la universidad (calidad del entorno de aprendizaje y aspectos de bienestar, como el alojamiento para estudiantes).

- Las características personales del alumno.

- Condiciones externas a la universidad, como la situación financiera y si el estudiante trabajaba a tiempo parcial mientras estudiaba.

- Condiciones de estudio en la universidad. En este punto destacan los recursos institucionales, como el número de estudiantes por asignatura; nivel de calificación del personal; intensidad de la investigación; proporción personal general-estudiantes, gasto académico por estudiante, gasto de biblioteca por alumno; el plan de estudio; la calidad del entorno de aprendizaje, y los servicios de apoyo y asesoramiento en las decisiones de abandono; los pares o compañeros de estudio, y el tema de estudio (particularmente los estudios relacionado a las ciencias).

Por otra parte, y como explican Larsen, Sommersel y Larsen (2013), el proceso de deserción podría explicarse por situaciones originadas en tres periodos o fases: la preuniversitaria, la intrauniversitaria y la de toma de decisiones. En la fase preuni- 
versitaria intervendrían factores socioeconómicos, los prerrequisitos de estudio y la elección de la carrera o universidad. Luego, en la fase intrauniversitaria, las razones que explicarían la deserción se relacionan al potencial de éxito, las motivaciones de estudio, la integración en la universidad (tanto académica como socialmente), las condiciones de estudio, y la existencia de servicios de apoyo. Por último, en la fase de toma de decisiones estarían las razones relacionadas a la situación económica, las condiciones de vida (como la situación familiar, enfermedades y situación de vivienda), los servicios de asesoramiento (que pueden provenir de las autoridades educativas, de la familia o amigos), y los planes de futuro relacionados a la búsqueda de nuevos trabajos, carreras o universidades.

En definitiva, son pocas las razones por las cuales las políticas universitarias pueden influir en la decisión de abandono de los estudiantes, y estas son principalmente las relacionadas a las condiciones de estudio de los estudiantes, es decir, las condiciones institucionales para estudiar, la calidad docente, los servicios de apoyo y el nivel de dificultad y carga académica (Kehm, Larsen y Sommersel, 2020; Larsen, Sommersel y Larsen, 2013). Asimismo, el estudio de Canales y De los Ríos (2007) destacó que factores como la mala calidad de las instituciones universitarias, la infraestructura, calidad de profesorado o mallas curriculares, tenían un impacto en la deserción de los estudiantes que tenían mejores pruebas de ingreso.

Otro factor institucional analizado ha sido la biblioteca. Kramer y Kramer (1968) demostraron que existe una correlación estadísticamente significativa entre el uso de la biblioteca (préstamo de libros) y la persistencia entre los estudiantes de primer año. Además, Mezick (2007) expuso que los gastos de la biblioteca y el personal profesional tienen un efecto positivo y significativo en la retención de estudiantes; a mayor interacción entre los bibliotecarios y la comunidad universitaria mayor será el impacto positivo en la vida de los estudiantes. Por otro lado, Soria, Fransen y Nackerud (2017) indican que el uso de la biblioteca al menos una vez durante el primer año, junto al uso de recursos electrónicos y libros, aumenta significativamente las probabilidades de graduarse en cuatro años sobre el retiro de la universidad.

Otras investigaciones se enfocaron a detectar elementos que llevan a persistir en los estudios universitarios. Una de ellas es la de Müller y Braun (2018), que detectó que los egresados consideraban que factores importantes para tener éxito en sus estudios fueron el trabajo colaborativo junto a otros estudiantes, haber estructurado su aprendizaje de forma independiente y el contacto con los profesores. Sobre este último punto, Dwyer (2017) demostró que las interacciones entre estudiantes y profesores son un componente de la integración social que puede generar persistencia y beneficios académicos; además, esta interacción se asocia con altos niveles de compromiso educativo. Por otra parte, Lerdpornkulrat, Koul y Poondej (2018) demostraron que las percepciones del clima del aula y factores institucionales-como estructuras y metas institucionales- están asociadas significativamente con las orienta- 
ciones motivacionales de los estudiantes y sus niveles de participación. En conjunto, estas percepciones contribuyen a la intención de persistir en la universidad y a la identificación institucional.

Finalmente, dos elementos que ayudan a persistir en los estudios son el apoyo emocional y económico que reciben los estudiantes de sus familiares, amigos y compañeros, junto a las ayudas económicas (Facchini, Triventi y Vergolini, 2020; Gündüz y Karaman, 2020).

\section{Metodología}

\section{Objetivo}

El objetivo de esta investigación es identificar y analizar los factores institucionales que explican la deserción estudiantil de las universidades chilenas. Para su cumplimiento, en una primera etapa se hizo necesario detectar, por medio de una revisión bibliográfica, los distintos factores que podrían explicar la tasa de retención y deserción de los estudiantes universitarios, desde la perspectiva institucional. Igualmente fue necesario determinar los factores institucionales a analizar y se aplicó la técnica de panel de datos para determinar cómo inciden en la tasa de deserción universitaria. Finalmente, y a partir de lo anterior, se interpretaron los resultados obtenidos.

\section{Tipo de diseño}

Se llevó a cabo un estudio exploratorio, de carácter cuantitativo, con aplicación de técnicas de datos de panel, combinando tanto una dimensión de orden transversal como temporal. Para el tratamiento de los datos se utilizó el software Stata.

\section{Hipótesis}

En virtud del objetivo de investigación y del marco teórico que la sustenta, se plantean las siguientes hipótesis:

H1: A menor número de estudiantes por docentes con grado de doctor, menor es la tasa de deserción estudiantil.

H2: A mayor cantidad de profesores con postgrado, menor es la tasa de deserción estudiantil.

H3: A mayor cantidad de metros cuadrados construidos de salas de estudio, laboratorios y salas de clases por estudiante, menor es la tasa de deserción estudiantil.

H4: A mayor número de laboratorios, salas de estudios y salas de clases, menor es la tasa de deserción estudiantil.

H5: A mayor cantidad de metros cuadrados construidos de bibliotecas por estudiantes, menor es la tasa de deserción.

H6: A mayor número de bibliotecas académicas, menor es la tasa de deserción. 


\section{Muestra}

El estudio se realizó utilizando una muestra de 28 universidades chilenas, de las cuales 12 son privadas y 16 estatales. Cada una de estas instituciones cumple con altos estándares de calidad, según la acreditación que han obtenido. El periodo analizado es de tres años, comprendido entre el 2016 y el 2018.

\section{Procedimiento de análisis}

Metodológicamente, y en coherencia con el objetivo de esta investigación, se determinó como variable dependiente la tasa de deserción (deserción), medida a partir de la tasa de retención de cada universidad, informada por el Consejo Nacional de Educación del Gobierno de Chile (Consejo Nacional de Educación, 2020).

Luego, las variables independientes fueron cantidad de estudiantes por académico con grado de doctor (estbydoc), número de académicos con postgrado (graduates); metros cuadrados construidos de salas de estudio, laboratorios, salas de clase por estudiantes (squaremt); cantidad total de salas de estudio, laboratorios y salas de clases (nlabsalas); número total de bibliotecas académicas (nbib), y cantidad de metros cuadrados de biblioteca por estudiantes (bibsqmt).

A partir de la información recopilada relacionada a las variables dependiente e independientes, en una primera etapa se hizo un análisis descriptivo de las variables bajo estudio. Luego, en una segunda etapa se realizó un estudio de tipo correlacional y no experimental, que evaluó la relación entre los factores institucionales de las universidades y la tasa de deserción estudiantil, sin manipular las variables. Finalmente se hicieron tres regresiones de tipo panel de datos: OLS Pooler, Efectos fijos y Efectos aleatorios. Dado que se detectó la presencia de heterocedasticidad, autocorrelación de orden 1 y correlación contemporánea, se incorporó una estimación robusta y mediante el estimador de White se llegó al modelo final. El software estadístico utilizado fue Stata.

Los modelos a estimar son los siguientes:

\section{OLS Pooled:}

$$
\begin{aligned}
& \text { Deserción }_{i t}=\beta_{0}+\beta_{1} \text { estbydoc }_{i t}+\beta_{2} \text { sraduates }_{i t}+\beta_{3} \text { squaremt }_{i t} \\
& +\beta_{4} \text { nlabsalas }_{i t}+\beta_{5} \text { bbib }_{i t}+\beta_{6} \text { bibsqmt }_{i t}+\varepsilon_{i t} \\
& i=\underline{1,28}, t=\underline{1,3} \varepsilon_{i t} \sim N\left(0 ; \sigma_{\varepsilon}^{2}\right)
\end{aligned}
$$

\section{FE: Efectos fijos:}

$$
\begin{aligned}
& \text { Deserción }_{i t}=(\alpha+\gamma) \beta_{0}+\beta_{1} \text { estbydoc }_{i t}+\beta_{2} \text { sraduates }_{i t}+\beta_{3} \text { squaremt }_{i t} \\
& +\beta_{4} \text { nlabsalas }_{i t}+\beta_{5} \text { nbib }_{i t}+\beta_{6} \text { bibsqmt }_{i t}+\mathrm{e}_{i t} \\
& i=\underline{1,28}, t=\underline{1,3} \varepsilon_{i t} \sim N\left(0 ; \sigma_{\varepsilon}^{2}\right)
\end{aligned}
$$




\section{RE: Efectos aleatorios:}

$$
\begin{aligned}
& \text { Deserción }_{i t}=\alpha+\beta_{1} \text { estbydoc }_{i t}+\beta_{2} \text { sraduates }_{i t}+\beta_{3} \text { squaremt }_{i t}+\beta_{4} \text { nlabsalas }_{i t} \\
& +\beta_{5} \text { nbib }_{i t}+\beta_{6} \text { bibsqmt }_{i t}+\left(u_{i}+v_{i t}\right) \\
& i=\underline{1,28}, t=\underline{1,3} \varepsilon_{i t} \sim N\left(0 ; \sigma_{\varepsilon}^{2}\right)
\end{aligned}
$$

Donde:

- Deserción corresponde a la tasa de deserción, variable que toma valores entre 0 y 1 , y se calcula como 1 - tasa de retención.

- Estbydoc es la cantidad de estudiantes por académico con grado de doctor.

- Graduates es el número de académicos con postgrado.

- Squaremt es la cantidad de metros cuadrados de salas de estudio, laboratorios y salas de clases por estudiante.

- Nlabsalas es la cantidad total de salas de estudio, laboratorios y salas de clases.

- Nbib es la cantidad total de bibliotecas.

- Bibsqmt es la cantidad de metros cuadrados de bibliotecas por estudiante.

Además, $\beta_{0}$ es el intercepto para la regresión OLS Pooled, los betas $\beta_{1}, \beta_{2}, \beta_{3}$, $\beta_{4}, \beta_{5}, \beta_{6}$ son los efectos marginales de las variables correspondientes, $\alpha$ es el intercepto general para todas las universidades en todos los años (efectos fijos), $\gamma_{i}$ es el efecto individual del individuo $i$ (efectos fijos), $u_{i}$ es la heterogeneidad individual no-observada (efectos aleatorios) y $\varepsilon_{i t}$ es el error aleatorio (todos los modelos). Los modelos robustos se han estimado mediante el estimador de White para la matriz de covarianzas:

$$
\hat{\Sigma}_{\text {white }}=\left(X^{\prime} X\right)^{-1}\left(X^{\prime} \operatorname{diag}\left(\hat{u}_{1}^{2}, \ldots, \hat{u}_{n}^{2}\right) X\right)\left(X^{\prime} X\right)^{-1}
$$

La información utilizada correspondió a datos obtenidos de las universidades pertenecientes al Consejo de Rectores de Universidades Chilenas (CRUCH). Para ello se recurrió a los datos entregados por el Servicio de Información de Educación Superior (SIES) y el Consejo Nacional de Educación (CNED). Se extrajo información de las 30 universidades adscritas al CRUCH para el periodo 2016 a 2018. Sin embargo, como dos universidades iniciaron sus actividades en el año 2017 (Universidad de Aysén y Universidad de O’Higgins), no fueron incluidas en el estudio, por lo tanto la muestra incluyó 28 instituciones, de ellas 16 son estatales y 12 son privadas.

\section{Resultados}

A partir de los datos obtenidos se observa que, para el conjunto de universidades de la muestra, la tasa de deserción promedio es de 18,5\%, con un mínimo de 10,5\% y un máximo de 25,1\% (tabla 1 ). 
Tabla 1. Resumen de los indicadores promedio de las universidades del CRUCH.

\begin{tabular}{|c|c|c|c|c|c|c|c|}
\hline Universidad & deserción & estbydoc & graduates & squaremt & nlabsalas & nbib & bibsqmt \\
\hline \multicolumn{8}{|c|}{ Todas las universidades } \\
\hline Media & $18.5 \%$ & 11.80 & 674.70 & 12.30 & 580.59 & 8.61 & 2.23 \\
\hline Mínimo & $10.5 \%$ & 4.44 & 135 & 3.13 & 118 & 1 & 0.78 \\
\hline Máximo & $25.1 \%$ & 19.9 & 2240 & 48.2 & 1917 & 48 & 6.11 \\
\hline Desviación estándar & 0.04 & 3.94 & 507.26 & 8.56 & 445.80 & 9.70 & 1.22 \\
\hline \multicolumn{8}{|c|}{ Universidades públicas } \\
\hline Media & $19.2 \%$ & 13.19 & 530.80 & 14.00 & 524.00 & 9.04 & 2.13 \\
\hline Mínimo & $15.4 \%$ & 8.37 & 135 & 4.56 & 232 & 2.67 & 0.78 \\
\hline Máximo & $25.1 \%$ & 19.9 & 2032 & 48.2 & 1727 & 48 & 4.15 \\
\hline Desviación estándar & 0.04 & 4.02 & 457.71 & 10.59 & 379.99 & 11.26 & 0.91 \\
\hline \multicolumn{8}{|c|}{ Universidades privadas } \\
\hline Media & $17.7 \%$ & 10.06 & 854.58 & 10.18 & 651.33 & 8.01 & 2.37 \\
\hline Mínimo & $10.5 \%$ & 4.44 & 367 & 3.13 & 118 & 1 & 1 \\
\hline Máximo & $23.8 \%$ & 15.3 & 2240 & 16.3 & 1917 & 22.7 & 6.11 \\
\hline Desviación estándar & 0.04 & 3.21 & 527.28 & 4.64 & 525.49 & 7.55 & 1.58 \\
\hline
\end{tabular}

Fuente: Elaboración propia.

Luego, en lo referido a los indicadores institucionales, el promedio de estudiantes por académico con grado de doctor es de 12 (D.E. = 4,2), con un rango entre 4.4 y 20.5 estudiantes y, en promedio, las universidades tienen 655 académicos con postgrado, fluctuando entre 133 y 2240. Con respecto a los indicadores de infraestructura, se ha reportado que en promedio las salas de estudio, laboratorios y salas de clase totalizan $12,1 \mathrm{~m}^{2}$ por estudiante (D.E. $=8,4$; rango: 3, 1-48, 8); el número de laboratorios y salas es de 566 (D.E. = 444,5; rango: 118-1917), hay 8,48 de bibliotecas (D.E. = 9,5; rango: 1-48) y 2,22 $\mathrm{m}^{2}$ de bibliotecas por estudiante (D.E. = 1.19; rango: 0.78-6.11).

Posteriormente, sobre la base de la clasificación de las universidades según su propiedad, se realizó un análisis de comparación de medias con base en una prueba T Student, definiéndose un nivel de significancia de $\alpha=0,05$ (tabla 2). Los resultados indican que la tasa de deserción es mayor en promedio en $2,8 \%$ en las universidades estatales, siendo evidencia estadísticamente significativa ( $p=0,002)$; así también, la cantidad de estudiantes por académico con grado de doctor es mayor en 3,2 estudiantes en las entidades del estado ( $\mathrm{p}<0,001)$; por su parte, la cantidad de académicos con postgrado es superior en 289 docentes en las universidades no estatales ( $p=0,008)$. No se han reportado diferencias significativas en los metros cuadrados totales $(\mathrm{p}=$ $0,251)$, en el total de salas y laboratorios ( $p=0,113)$, en el número de bibliotecas ( $p$ $=0,139)$ ni en la superficie de bibliotecas por estudiante $(\mathrm{p}=0,337)$. 
Tabla 2. Medias por indicador y prueba T de Student.

\begin{tabular}{ccccc}
\hline Indicador & $\begin{array}{c}\text { Estatal } \\
(\mathrm{n}=16)\end{array}$ & $\begin{array}{c}\text { No estatal } \\
(\mathrm{n}=12)\end{array}$ & $T$ & $P$ \\
\hline deserción & $19,9 \%$ & $17,1 \%$ & $3,17^{* *}$ & 0,002 \\
\hline estbydoc & 13,5 & 10,3 & $3,64^{* * *}$ & $<0,001$ \\
\hline graduates & 531,5 & 820,6 & $-2,70^{* *}$ & 0,008 \\
\hline squaremt & 13,0 & 10,8 & 1,16 & 0,251 \\
\hline nlabsalas & 499,4 & 654,1 & $-1,60$ & 0,113 \\
\hline nbib & 9,8 & 6,6 & 1,49 & 0,139 \\
\hline bibsqmt & 2,1 & 2,4 & $-0,97$ & 0,337 \\
\hline
\end{tabular}

Fuente: Elaboración propia.

Al hacerse un análisis por año, se observa que para el año 2016 la tasa de deserción promedio fue de 19,4\%, disminuyendo a 17,1\% en el año siguiente y finalmente para el año 2018 volvió a aumentar a 19,6\%. En términos académicos, la cantidad de estudiantes por académico doctor fluctuó entre 11,9 y 12,2 estudiantes, mientras que la cantidad de académicos con grado de magíster o doctor pasó de 634 docentes en el 2016 a 676 docentes en el 2018, es decir, aumentó 6,6\%.

En cuanto a los indicadores de infraestructura, la cantidad de $\mathrm{m}^{2}$ totales por estudiante fluctuó entre 11,6 y 12,9 m², no modificándose de manera sustancial; la cantidad total de laboratorios y salas pasó de 536 en el 2016 a 589 en el 2018, aumentando en 9,9\%. Finalmente, el número medio de bibliotecas ha ido disminuyendo paulatinamente, pasando de 8.63 en el 2016 a 8.37 en el 2018, mientras que la superficie media de bibliotecas por estudiante varió entre 2,17 y 2,27 $\mathrm{m}^{2}$. En definitiva, se puede observar que los indicadores obtenidos para los factores institucionales en general se mantienen a lo largo de los años, mientras que otros como la cantidad de estudiantes por doctor, el número total de salas, laboratorios y bibliotecas van fluctuando de forma creciente o decreciente.

Tabla 3. Medias por indicador, por año.

\begin{tabular}{cccc}
\hline Indicador & Año 2016 & Año 2017 & Año 2018 \\
\hline deserción & $19,4 \%$ & $17,1 \%$ & $19,6 \%$ \\
\hline estbydoc & 12,1 & 11,9 & 12,2 \\
\hline graduates & 634 & 655 & 676 \\
\hline squaremt & 11.7 & 12.9 & 11.6 \\
\hline nlabsalas & 536 & 571 & 589 \\
\hline nbib & 8.63 & 8.44 & 8.37 \\
\hline bibsqmt & 2.23 & 2.27 & 2.17 \\
\hline
\end{tabular}

Fuente: Elaboración propia. 


\section{Análisis de correlación}

En esta segunda sección se ha estudiado el nivel de relación que tienen las variables seleccionadas. Para esto se ha aplicado correlación de Pearson para las variables, obteniendo los resultados que se muestran en la tabla 4. Por un lado, a mayor número de estudiantes por académico doctor, mayor tasa de deserción $(r=0.352, p<0.01)$. Por otro lado, a mayor número de académicos con grado $(\mathrm{r}=-0.367, \mathrm{p}<0.001)$, a mayor cantidad de superficie total de salas y laboratorios $(\mathrm{r}=-0.284$, $\mathrm{p}<0.01$ ), a mayor cantidad de salas $(\mathrm{r}=-0.269, \mathrm{p}<0.01)$ y a mayor cantidad de superficie de bibliotecas por alumno $(\mathrm{r}=-0.515, \mathrm{p}<0.001)$, menor será la tasa de deserción, todas estas son estadísticamente significativas.

Tabla 4. Matriz de correlaciones para indicadores.

\begin{tabular}{|c|c|c|c|c|c|c|c|}
\hline Indicador & deserción & estbydoc & graduates & Squaremt & nlabsalas & nbib & bibsqmt \\
\hline deserción & - & & & & & & \\
\hline estbydoc & $0.35^{* *}$ & - & & & & & \\
\hline graduates & $-0.37 * * *$ & $-0.46^{* *}$ & - & & & & \\
\hline squaremt & $-0.284^{* *}$ & -0.09 & 0.114 & - & & & \\
\hline nlabsalas & $-0.27^{*}$ & $-0.261 *$ & $0.91 * * *$ & $0.25^{*}$ & - & & \\
\hline nbib & -0.08 & $-0.27^{*}$ & $0.68^{* * *}$ & 0.12 & $0.70^{* * *}$ & - & \\
\hline bibsqmt & $-0.52 * * *$ & $-0.24 *$ & $0.41 * * *$ & $0.26 *$ & $0.36^{* *}$ & 0.18 & - \\
\hline
\end{tabular}

Significancia $=* \mathrm{p}<0,05 ; * * \mathrm{p}<0,01 ; * * * \mathrm{p}<0,001$.

Fuente: Elaboración propia.

\section{Análisis de regresiones}

Como se muestra en la tabla 5, el modelo OLS Pooled inicial detectó un efecto negativo y estadísticamente significativo para el número de graduados ( $\mathrm{p}<0.05)$, la superficie total de salas y laboratorios por estudiante $(\mathrm{p}<0.05)$ y la superficie total de bibliotecas por estudiante $(\mathrm{p}<0.01)$, explicando $<39.9 \%$ de la variación de la tasa de deserción. Las pruebas de heteroscedasticidad y autocorrelación indicaron incumplimiento de los supuestos, por lo cual se estimó la versión robusta. En esta versión se mantienen las conclusiones, añadiendo el efecto positivo y significativo del número de bibliotecas $(\mathrm{p}<0.001)$ y con una mayor significancia para la superficie total de bibliotecas por estudiante $(\mathrm{p}<0.001)$. 
Tabla 5. Estimaciones de los modelos propuestos.

\begin{tabular}{|c|c|c|c|c|c|c|c|}
\hline \multirow[b]{2}{*}{ Variable } & \multirow{2}{*}{$\begin{array}{c}\text { Resultado } \\
\text { esperado }\end{array}$} & \multicolumn{3}{|c|}{ Estimaciones iniciales } & \multicolumn{3}{|c|}{ Estimaciones robustas } \\
\hline & & Pooled & $\mathrm{FE}$ & RE & Pooled & $\mathrm{FE}$ & RE \\
\hline estbydoc & + & 0.001026 & 0.003464 & 0.0016666 & 0.001026 & $0.003464 *$ & 0.001666 \\
\hline graduates & - & $-0.000046^{*}$ & 0.000022 & -0.0000202 & $-0.000046^{*}$ & 0.000022 & -0.000020 \\
\hline squaremt & - & $-0.001077 *$ & -0.000937 & -0.0010520 & $-0.001077 * *$ & -0.000937 & $-0.001052 *$ \\
\hline nlabsalas & - & 0.000022 & -0.000077 & 0.0000002 & 0.000022 & -0.000077 & 0.0000002 \\
\hline nbib & - & 0.0011040 & -0.000998 & 0.0006117 & $0.001104 * * *$ & -0.000998 & 0.000612 \\
\hline bibsqmt & - & $-0.011750^{* *}$ & -0.052747 & $-0.0135923^{*}$ & $-0.011750 * * *$ & $-0.052747^{*}$ & $-0.013592 * *$ \\
\hline constante & & $0.221256^{* * *}$ & $0.309775^{* * *}$ & $0.2154086^{* * *}$ & $0.2212562^{* * *}$ & $0.309775^{* * *}$ & $0.215409^{* * *}$ \\
\hline Het.Test & & & $13.85^{* * *}$ & $10.73^{* * *}$ & & & \\
\hline Autocorr & & $15303 * * *$ & $5.20 *$ & $3.87 *$ & & & \\
\hline Correl. cont. & & $15.505^{2}$ & $2.897 * *$ & $6.636^{* * *}$ & & & \\
\hline $\mathrm{n}$ & & 81 & 81 & 81 & 81 & 81 & 81 \\
\hline $\mathrm{R}^{2}$ & & 0.39865 & 0.15987 & & 0.39865 & 0.15987 & \\
\hline $\mathrm{R}^{2}$ Overall & & & 0.29127 & 0.38225 & & 0.29127 & 0.38225 \\
\hline $\mathrm{R}^{2}$ Between & & & 0.35300 & 0.47312 & & 0.35300 & 0.47312 \\
\hline $\mathrm{R}^{2}$ Within & & & 0.15987 & 0.06815 & & 0.15987 & 0.06815 \\
\hline$\sigma_{u}^{2}$ & & & 0.07104 & 0.02683 & & 0.07104 & 0.02683 \\
\hline$\sigma_{\varepsilon}^{2}$ & & & 0.02332 & 0.02332 & & 0.02332 & 0.02332 \\
\hline$\rho$ & & & 0.90274 & 0.56972 & & 0.90274 & 0.56972 \\
\hline
\end{tabular}

Fuente: Elaboración propia.

Para la especificación con efectos fijos, no se observó ningún efecto de los indicadores, reportando solo 16\% de explicación de la variabilidad; sin embargo se detectó heteroscedasticidad, teniendo que estimar la versión robusta. En esta versión se detectó un efecto positivo y estadísticamente significativo para la razón del número de estudiantes por doctor ( $\mathrm{p}<0.05$ ), y a su vez un efecto negativo para la superficie total de bibliotecas por estudiante $(\mathrm{p}<0.05)$.

Para la especificación con efectos aleatorios, solo se observó un efecto negativo y estadísticamente significativo para la superficie total de bibliotecas por estudiante ( $\mathrm{p}$ $<0.05)$, pero, al igual que en caso anterior, se detectó heteroscedasticidad, estimando de igual forma la versión robusta. En esta estimación se ha añadido como significativo el efecto de la superficie total de salas y laboratorios por estudiante $(p<0.05)$.

En los tres modelos precedentes se realizaron pruebas para evaluar el cumplimiento de supuestos con base en varianza y autocorrelación, además de la correlación contemporánea en los modelos de panel (fijo y aleatorio). En todos los casos no se pudo evidenciar el cumplimiento de los supuestos $(\mathrm{p}<0.05)$. En definitiva, para aplacar estos tres inconvenientes de forma simultánea, se ajustó el modelo final con base en el estimador que corrige simultáneamente estos (xtpcse en Stata). 
En definitiva, el modelo final incorporó una estimación robusta de forma simultánea ante la presencia de heteroscedasticidad, autocorrelación de orden 1 y correlación contemporánea. A partir de él se determinó que hay efectos negativos y estadísticamente significativos para el número de graduados $(\mathrm{p}<0.05)$, la superficie total de salas y laboratorios por estudiante $(p<0.05)$ y la superficie total de bibliotecas por estudiante $(\mathrm{p}<0.05)$. Se ha obtenido un efecto positivo para el número de bibliotecas ( $p<0.001$ ). Este modelo es capaz de explicar el $49.7 \%$ de la variabilidad de la tasa de deserción de las universidades seleccionadas. A partir de este resultado, es posible señalar el modelo estimado en la tabla 6.

Tabla 6. Modelo estimado.

\begin{tabular}{|c|c|c|c|}
\hline Variable & & Resultado esperado & Resultado obtenido \\
\hline estbydoc & & + & 0.0012193 \\
\hline graduates & & - & $-0.0000420^{* *}$ \\
\hline squaremt & & - & $-0.0011086^{* * *}$ \\
\hline nlabsalas & & - & 0.0000185 \\
\hline nbib & & - & $0.0010412^{* * *}$ \\
\hline bibsqmt & & - & $-0.0123320 * * *$ \\
\hline constante & & & $0.2213393^{* * *}$ \\
\hline \multicolumn{4}{|c|}{$\begin{array}{ll}\mathrm{n} & 81\end{array}$} \\
\hline \multicolumn{4}{|c|}{$\mathrm{R}^{2} \quad .049720$} \\
\hline \multicolumn{4}{|c|}{$\rho \quad 0.20591$} \\
\hline
\end{tabular}

Fuente: Elaboración propia.

\section{DisCUSIÓN Y CONCLUSIONES}

\section{Discusión}

La deserción de estudiantes universitarios se puede deber a múltiples factores, pudiendo ser voluntarios o involuntarios. Cualquiera sea la causa que lo origine, la deserción tiene implicancias no solo para los estudiantes que hacen abandono de sus estudios sino también para sus familias, para las instituciones y para la sociedad, en general. Y esto no se debe únicamente a los recursos invertidos en el alumno que deserta, porque la educación superior aporta a las personas elementos y posibilidades indispensables para el desarrollo personal y laboral, y de la sociedad en su conjunto. La educación superior permite que las personas se inserten en el mercado laboral en condiciones más favorables, permitiendo así una mayor equidad social. Por otro lado, la deserción conlleva a que se perpetúen situaciones de exclusión social y económica.

En cuanto a las instituciones, la deserción no solo afecta el cumplimiento de sus misiones, sino que también impacta en sus ingresos e índices de eficiencia y calidad, 
por lo tanto, es necesario e importante que se tomen medidas para disminuir la deserción estudiantil. Si bien hay factores que favorecen la deserción y son ajenos a las instituciones de educación superior, hay otros en los que sí pueden influir por medio de sus políticas internas. De hecho, las universidades han definido programas y planes que buscan generar las condiciones institucionales para una mayor retención de los estudiantes, especialmente en los primeros años.

Esta investigación tenía por finalidad identificar y analizar los factores institucionales que explican la deserción estudiantil de las universidades chilenas. Por medio de una revisión bibliográfica se identificaron aspectos sobre los que las universidades pueden influir; entre ellos estarían los recursos institucionales, como el número de estudiantes por asignatura; nivel de calificación del personal; intensidad de la investigación; proporción personal general-estudiantes, gasto académico por estudiante, gasto de biblioteca por alumno y la calidad del entorno de aprendizaje, entre otros.

De acuerdo a las hipótesis planteadas, se esperaba que todas las variables concernientes a la infraestructura impactaran negativamente en la tasa de deserción. En efecto, en este aspecto se evaluaron distintas dimensiones, como son las bibliotecas y las salas de estudio, laboratorios y salas de clases, medidos ambos por los metros cuadrados por estudiante y cantidad de ellos. La razón que debería llevar a tener una relación negativa es que estos espacios no solo permiten que se desarrollen en su interior las actividades propias de la docencia y aprendizaje de los estudiantes, sino que también permiten que exista una mayor y mejor interacción de los estudiantes con sus docentes y entre ellos.

Igualmente se consideraron variables relacionadas a la calidad académica de la universidad: número de académicos con postgrado y cantidad de estudiantes por académico con grado de doctor. Mientras se esperaba que el número de académicos con postgrado impactara negativamente en la tasa de deserción, se esperaba que la cantidad de estudiantes por académicos con grado de doctor tuviese un impacto positivo.

\section{Conclusiones}

En definitiva, los hallazgos señalaron que las universidades privadas tienen una menor deserción que las estatales. Además, para el conjunto de universidades, se constató que cuatro variables impactaban significativamente en la tasa de deserción universitaria: la cantidad de metros cuadrados de bibliotecas por estudiante; la cantidad de metros cuadrados de salas de estudio, laboratorios y salas de clases por estudiante; la cantidad total de bibliotecas, y el número de académicos con postgrado. De ellas, las tres primeras corresponden a factores relacionados con la infraestructura de las universidades. 
De acuerdo a las hipótesis 5 y 3 , respectivamente, tanto la cantidad de metros cuadrados por estudiante destinados a las bibliotecas como a las salas de estudio, laboratorios y salas de clases debían impactar negativamente en la tasa de deserción. Los resultados evidenciaron que, efectivamente, estas variables tienen un efecto significativo y negativo en la variable dependiente, lo que permite aceptar ambas hipótesis.

Los resultados esperados sobre el efecto que tendría la cantidad de bibliotecas en la variable dependiente no fueron los esperados. De acuerdo a la hipótesis 6, la cantidad de bibliotecas debería impactar negativamente en la tasa de deserción, favoreciendo la retención de estudiantes. La explicación a esta relación negativa se debe a que un mayor número de bibliotecas entrega mayores posibilidades de acceder a ellas. Asimismo, podría ser asociado a una alta calidad de la infraestructura institucional. Sin embargo, se encontró una relación positiva y significativa, lo que no permitió aceptar la hipótesis 6 , por lo tanto se hace necesario indagar con mayor profundidad en los resultados obtenidos. Una explicación a la relación positiva encontrada podría ser que hoy los recursos bibliográficos se encuentran en las páginas web de las instituciones; igualmente cada día hay mayores posibilidades de acceder a libros y artículos disponibles en recursos digitales, haciendo que los estudiantes no requieran dirigirse a las bibliotecas.

Para conocer el efecto que tendría la calidad del cuerpo docente se utilizó la variable número de académicos con postgrado. De acuerdo a la hipótesis 2, a mayor cantidad de profesores con postgrado, menor debería ser la tasa de deserción estudiantil. Como se evidenció en el estudio, esta relación se da de manera significativa, lo que permite aceptar esta hipótesis. Por lo tanto, es posible inferir que la calidad del cuerpo docente debe ser una preocupación permanente de las universidades, que deben apoyar y motivar la inversión en capital humano, es decir, que sus académicos puedan lograr altos niveles de formación.

Por último, dos variables entregaron resultados no significativos: cantidad de estudiantes por académico con grado de doctor y cantidad total de salas de estudio, laboratorios y salas de clases. En la primera de ellas se encontró que la variable impactaba positivamente a la tasa de deserción. Esto estaba de acuerdo a lo planteado en la hipótesis 1, ya que, al haber un menor número de estudiantes por docente, se esperaría una mayor comunicación e interacción entre las partes, facilitando la integración social de los estudiantes y apoyando de mejor forma los procesos de enseñanza-aprendizaje. Sin embargo, esta relación no es significativa, por lo tanto no es posible aceptar la hipótesis 1.

En cuanto a la variable cantidad total de salas de estudio, laboratorios y salas de clases, nuevamente se esperaba una relación negativa con la variable dependiente. Los resultados mostraron que esta relación se daría, aunque de manera no significativa, haciendo que no se pueda aceptar la hipótesis 4. 
En definitiva, los resultados de esta investigación permiten describir el estado actual del nivel de deserción de las universidades chilenas en términos de factores institucionales, tomando como muestra las instituciones adscritas al Consejo de Rectores de Universidades Chilenas. Esto, en su conjunto, permite el desarrollo de políticas institucionales y públicas que ayuden a mejorar la retención de los estudiantes.

Este estudio abre la posibilidad de futuras investigaciones. Por una parte, sería interesante ampliar el alcance del estudio a un mayor número de universidades, sean chilenas o de otros países. Por otra, podrían ser incorporadas variables relacionadas a la calidad, como son los años de acreditación o resultado obtenido en los rankings internacionales, y variables asociadas al uso de las plataformas de aprendizaje y acceso a los recursos bibliográficos de manera remota.

Finalmente, se evidencia una limitación del estudio, esta es que se analizó el periodo 2016-2018, no pudiéndose incorporar los años 2019 y 2020. La razón es que a fines del año 2019 ocurrió en Chile el denominado "estallido social” que generó protestas y paros estudiantes, lo que pudo afectar la tasa de deserción. De igual forma, la pandemia producida por el Coronavirus (COVID-19) pudo afectar la tasa de inscripción y retención de los estudiantes universitarios en el año académico 2020. Asimismo, esta pandemia obligó a que las instituciones modificaran la modalidad de enseñanza, pasando de presencial a distancia.

\section{REFERENCIAS}

BCN [Biblioteca del Congreso Nacional de Chile] (2018). Guia legal sobre: educación superiory gratuidad. Recuperado de: https://www.bcn.cl/leyfacil/recurso/educacionsuperior-y-gratuidad.

Cabrera, L., Bethencourt, J., Alvarez, P., y González, M. (2006). El problema del abandono de los estudios universitarios. Relieve, 9, 103-134. Recuperado de: http://www.uv.es/RELIEVE/v12n2/ RELIEVEv12n2_1eng.htm.

Canales, A., y De los Ríos, D. (2007). Factores explicativos de la deserción universitaria. Calidad en la Educación, (26), 173. https://doi.org/10.31619/caledu.n26.239.

Consejo Nacional de Educación (2020). Índices educación superior. Recuperado de: https://www.cned.cl/indices/retencion-primer-ano.

CRUCH (2021). Consejo de Rectores de Universidades Chilenas. Recuperado de: https://www.consejoderectores.cl/.

Dwyer, T. (2017). Persistence in higher education through student-faculty interactions in the classroom of a commuter institution. Innovations in Education and
Teaching International, 54(4), 325-334. https:/ / doi.org/ 10.1080/14703297.2015.1112297.

Facchini, M., Triventi, M., y Vergolini, L. (2020). Do grants improve the outcomes of university students in a challenging context? Evidence from a matching approach. Higher Education. https://doi.org/10.1007/ s10734-020-00586-3.

González, L. E., y Espinoza, O. (2015). Deserción en educación superior en América Latina y el Caribe. Paideia, 45(jul.-dic.), 33-46. Recuperado de: http:// www.revistapaideia.cl/index.php/PAIDEIA/article/ view/71.

Gündüz, M., y Karaman, S. (2020). Open education faculty and distance education students' dropout reasons: The case of a Turkish State university. Open Praxis, 12(1), 7. https://doi.org/10.5944/openpraxis.12.1.970.

Himmel, E. (2002). Modelo de análisis de la deserción estudiantil en la educación superior. Calidad en la Educación, (17), 91. https://doi.org/10.31619/caledu. n17.409. 
Kehm, B. M., Larsen, M. R., y Sommersel, H. B. (2020). Student dropout from universities in Europe: A review of empirical literature. Hungarian Educational Research Journal, 9(2), 147-164. https://doi. org/10.1556/063.9.2019.1.18.

Kim, D., y Kim, S. (2018). Sustainable education: analyzing the determinants of university student dropout by nonlinear panel data models. Sustainability, 10(4), 954. https://doi.org/10.3390/su10040954.

Kramer, L. A., y Kramer, M. B. (1968). The College library and the drop-out. College \& Research Libraries, 29(4), 310-312. https://doi.org/10.5860/crl_29_04_310.

Larsen, M., Sommersel, H., y Larsen, M. (2013). Evidence on dropout phenomena at universities. Recuperado de: http:/ / edu.au.dk/fileadmin/edu/Udgivelser/Clearinghouse/ Review/Evidence_on_dropout_from_universities_ brief_version.pdf.

Lerdpornkulrat, T., Koul, R., y Poondej, C. (2018). Relationship between perceptions of classroom climate and institutional goal structures and student motivation, engagement and intention to persist in college. Journal of Further and Higher Education, 42(1), 102-115. https:/ / doi.org/10.1080/0309877X.2016.1206855.

Martí, M. L. S., Puertas, R. M., y Calafat, C. M. (2014). Calidad y eficiencia de las universidades públicas españolas. Revista de Estudios Regionales, 99, 135-154. http://www.scopus.com/inward/record.url?eid=2s2.0-84904673451\&partnerID=tZOtx3y1.

Mezick, E. (2007). Return on investment: Libraries and student retention. The Journal of Academic Librarianship, 33(5), 561-566. DOI: https://doi.org/10.1016/j.acalib.2007.05.002.

Mineduc (2016). Memoria Financiamiento Estudiantil 2016. Recuperado de: https://portal.beneficiosestudiantiles. $\mathrm{cl} /$ memorias.

Mineduc (2020). Informe matrícula 2020 en educación superior en Chile. Servicio de Información de Educación Su- perior, 1-10. Recuperado de: http://www.mifuturo.cl/ wp-content/uploads/2018/SIES/informe matricula 2018_sies.pdf?x78460.

Müller, L., y Braun, E. (2018). Student engagement: A concept for an evidence-based quality management of German higher education institutions. Zeitschrift Fur Eržiebungswissenschaft, 21(3), 649-670. https://doi.org/10.1007/s11618-017-0799-2.

Munizaga, F. R., Cifuentes, M. B., y Beltrán, A. J. (2018). Retención y abandono estudiantil en la educación superior universitaria en América latina y el Caribe. Education Policy Analysis Archives, 26, 1-29. https:/ / doi.org/10.14507/epaa.26.3348.

Rodríguez, M., y Zamora, J. (2014). Análisis de la deserción en la Universidad Nacional desde una perspectiva longitudinal. https://doi.org/10.13140/RG.2.2.30416.66569.

Salmi, J. (2014). Buenas prácticas internacionales de gobierno universitario. Nueva Revista, 116-132. Recuperado de: https://reunir.unir.net/handle/123456789/4431.

Smart, J. C. (1997). Higher education: Handbook of theory and research 12. Recuperado de: https://books.google. $\mathrm{cl} /$ books?id=twYjXLd6pyUC.

Soria, K. M., Fransen, J., y Nackerud, S. (2017). The impact of academic library resources on undergraduates' degree completion. College and Research Libraries, 78(6), 812-823. https://doi.org/10.5860/ crl.78.6.812.

Tapasco, O. A., Ruiz, F. J., Osorio, D., y Ramírez, D. (2019). Deserción estudiantil: incidencia de factores institucionales relacionados con los procesos de admisión. Educación y Educadores, 22(1), 81-100. https:// doi.org/10.5294/edu.2019.22.1.5.

Tinto, V. (1975). Dropout from higher education: A theoretical synthesis of recent research. Review of Educational Research, 45(1), 89-125. https://doi. org/10.3102/00346543045001089.

Cómo citar este artículo:

Sanhueza Gutiérrez, D., King-Domínguez, A., y Améstica-Rivas, L. (2021). Incidencia de la gestión universitaria en la deserción estudiantil de las universidades públicas en Chile. IE Revista de Investigación Educativa de la REDIECH, 12, e1270. doi: 10.33010/ie_rie_rediech.v12i0.1270. 